\title{
Design and Simulation of 3-DOF Reconfigurable Planar Parallel Robot
}

\author{
Zhili Hou ${ }^{1, a}$, Wenge $\mathrm{Wu}^{1}$, Ruiqin $\mathrm{Li}^{1}$ and Huibin Qin ${ }^{1}$ \\ ${ }^{1}$ School of Mechanical and Power Engineering, North University of China, 030051Taiyuan, China
}

\begin{abstract}
This paper presents the design approach for 3-DOF (degrees-of-freedom) reconfigurable planar parallel mechanism (RPPM) with modular kinematic limbs and hybrid platform. Using the proposed approach, a series of kinematic models with rotatability are obtained. At first, the main models of PPMs are constructed. Then a series of configuration models of PPM modules are reconstructed. And the new configuration families of 3-DOF PPMs are obtained. Finally, the motion trajectories of three driven layout configurations are simulated, and the results show that the hybrid-loop platform can expand the workspace of PPM. Especially, the configurations of the PPM can transform from the two operations modes to the original mode.
\end{abstract}

\section{Introduction}

In the past twenty years, researchers have made extensive research on reconfigurable mechanisms for its potential benefits and good application perspectives. Reconfigurable mechanisms can convert the original configuration from one to several new configurations in order to meet various task requirements [1]. The research involves the key problems such as configuration reorganization and reconstruction, singularity, controllability and so on.

Many application examples of reconfigurable parallel mechanism can be found in the recent studies [2-4] for both fields of machine tools [5-8] and industrial robotics [9-10]. And the endeffector of a manipulator is able to perform different kinds of motion.

A new reconstruction method for kinematic chain is the key step for the reconfigurable mechanism design. At present, the configuration reconstruction of parallel mechanism is mostly based on the diverse complex mathematical calculation, such as the screw theory [11], constraint changes characteristic of synthesis method [12], limb construction method [13], and so on. These calculation methods provide the algorithm rules for the configuration reconstruction.

In fact the concept of reconfigurable technology derived from the concept of modules as robotic cells [9]. For the design of most mechanical system, modular design has the advantages of high reliability and low cost, which is also suitable for the design of parallel mechanism [14].The parallel mechanisms are made of the same kinematic chains, and all the components used in each kinematic chain are the same [15].

3-DOF PPMs have the prominent characteristics, such as high rigidity, high precision and small workspace [16-17]. Because the traditional 3-DOF PPMs are not reconfigurable, the model design and

\footnotetext{
${ }^{a}$ Corresponding author : whitelily9988@163.com
} 
kinematic analysis of the eight structures have been studied from one type to another. Most researchers focus on characteristics analysis of one typical configuration for the PPMs [18-19]. In fact, the two or more types of the 3-DOF PPMs have the natural basis for reconfigurable configuration design. Moreover the eight configurations of the 3-DOF PPMs proposed in the paper can be reconfigured with some form of manual assistance. It is very important to find a unified method to realize its varying kinematics, and the modular units can quickly reconstruct new configurations.

Since the numbers of configurations are large, the configuration transformation efficiency should be improved. A new integrated modelling method is presented for the 3-DOF PPMs in this paper, which contains scale information and motion information of mechanism.

\section{Description of kinematic models}

A planar parallel robot consists of a moving platform and a fixed base which are connected to several kinematic limbs. There are two types of active joints used in planar parallel robots, namely, revolute $(\mathrm{R})$ and prismatic $(\mathrm{P})$. All the rotation axes of the revolute joints are parallel to the direction of the actuator. The axes between revolute pair and the prismatic pair are orthogonal to each other.

When the order of the kinematic pair $\mathrm{R}$ and $\mathrm{P}$ are changed, in accordance with the $\mathrm{R}$ and $\mathrm{P}$ of different combinations, eight planar kinematic chains having various structures are synthesized, they are RRR, RRP, RPR, RPP, PRR, PRP, PPR, PPP, but 3-PPP kinematic chain structure can't make the moving platform move, so the configuration is removed. Therefore, seven kinds of kinematic chains can be synthesized, the classification of planar kinematic chains are shown in table 1.

Table 1. The classification of planar kinematic chains.

\begin{tabular}{|c|c|}
\hline Number of prismatic pair & Types \\
\hline 0 & RRR \\
\hline 1 & RRP、RPR、PRR \\
\hline 2 & RPP、PRP、PPR \\
\hline
\end{tabular}

The structure of planar kinematic chain is completely symmetrical for the 3-DOF PPM. In order to meet the requirements of the symmetrical workspace, the kinematic pair ( $\mathrm{R}$ or $\mathrm{P}$ ) near to the fixed platform is selected as the actuator. In accordance with the joint position of the driven pair, eight planar kinematic chains are classified, as shown in Table 2.

Table 2. Eight kinematic chains of 3-DOF PPM.

\begin{tabular}{|c|c|c|c|c|c|c|c|c|}
\hline Actuator & \multicolumn{7}{|c|}{ Types } & Remark \\
\hline Joint 1 & $\underline{R R R}$ & $\underline{R} R P$ & $\underline{R P R}$ & $\underline{\text { PRR }}$ & & $\underline{\mathrm{PRP}}$ & $\underline{P P R}$ & \multirow{2}{*}{$\begin{array}{l}\text { The underlined letter } \\
\text { indicate the position of } \\
\text { actuated joint }\end{array}$} \\
\hline Joint 2 & & & $\mathrm{RPR}$ & & $\mathrm{RPP}$ & & & \\
\hline
\end{tabular}

\section{Design methodology}

\subsection{Full information modeling method}

Variable topology mechanism can realize special motion trajectories by changing topologized structure of the mechanism. Based on top-to-down design process, the full information models of PPMs are established, and the design process as shown in Figure 1. 


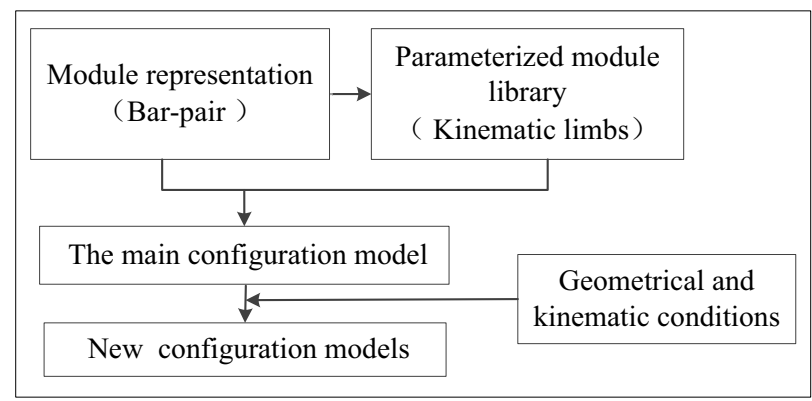

Figure.1 The top-to-down design flowchart

The full information model of a mechanism contains scale information and kinematic information. Scale information refers to the kinematic scale parameter (bar length, twist angle, axial length) and geometric constraint (co-axially, coplanar, parallel, etc.). Kinematic information includes the input and output motion parameters of PPMs. The full information models of the mechanism include main model and configuration rules. The main model contains full parameter model of the standard template.

Selecting a configuration of the PPMs as main model, a new family of PPMs and their configuration models are generated through topological transformation. It can be divided into three aspects to explain the topological transformation.

- The variable driven layout is changing position of the actuated pair through the hybrid platform.

- The variable parameter configuration is using the similar geometric features of configuration models to achieve a series of configuration models of PPMs.

- The variable kinematic chain is changing the modules of kinematic chains to construct new kinematic chains.

These transformation methods in the reconfiguration design reflect the relationship between the scale and motion constraints. The motion information can be mapped to the scale information.

\section{2 Configuration modules}

In order to design the RPPM models properly, PPM can divide into five modules, such as the input module, kinematic limb module, connecting layer module, support module and output module. There are several design rules should be required as follows:

When the constraint relationships of the main parameters are established, series of configuration model library are generated for the PPM. Take the bar-pair model as an example, the scale information models of planar bar-pair are constructed, as shown in Figure 2.In order to avoid motion interference phenomenon, the joint between bar-pair models should be designed as layer arrangement assembly.

Three-dimensional planar kinematic chains are constructed, as shown in Figure 3. 3-DOF planar parallel robot can be reconstructed with eight kinematic chains models, as shown in Figure 4.

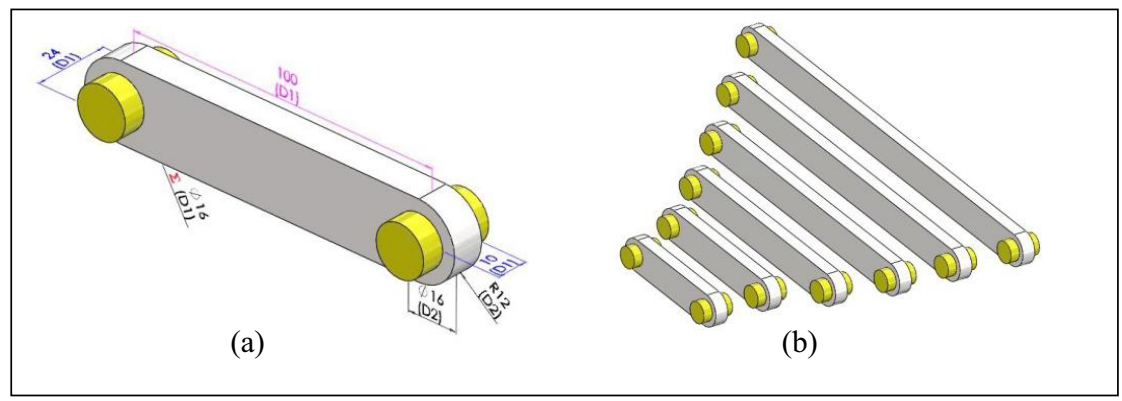

Figure.2 Models of planar bar-pair: (a) bar-pair model, and (b) serial bar-pair configuration models. 


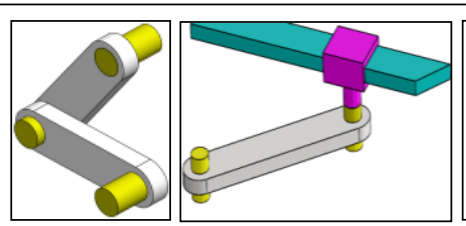

(a)

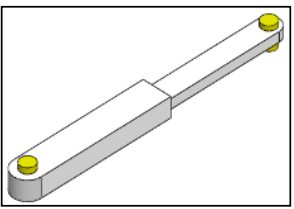

(c)

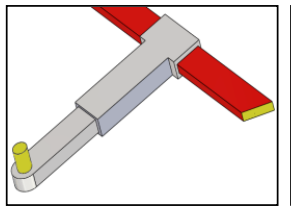

(d)

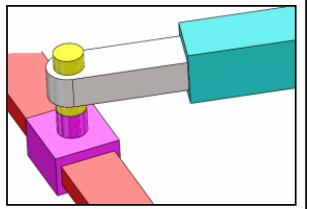

(e)

Figure.3 Models of planar kinematic chains: (a)RRR, (b)RRP/PRR, (c)RPR, (d)RPP/PPR, and (e)PRP.

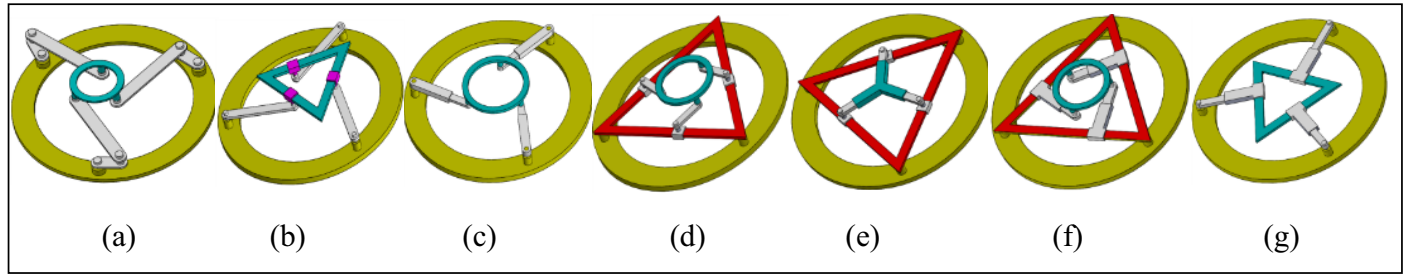

Figure.4 Models of 3-DOF PPM: (a)3-RRR,(b)3-RRP,(c)3-RPR or 3-RPR,(d) 3-PRR,(e) 3-PRP,(f) 3-PPR, and (g) 3-RPP.

\section{Design case}

When the driven layout of a mechanism is transformed, the different workspace of the mechanism can be obtained. Parallel hybrid platform is a new way to change the driven layout. If the parallel hybrid platform is established, the narrow workspace of parallel mechanism can be expanded.

Take the 3-RRR parallel mechanisms as an example, we establish the mixed orthogonal fixed platform and triangular fixed platform, as shown in Figure 5. When the joint $\mathrm{P}$ in the fixed platform is auto-locked in appropriate position, we can achieve different architectures of 3-RRR PPMs. The two modes (Figure 5) can also be swiched to the original mode ( Figure 4(a)). When the joint P (Figure 5) is auto-locked in appropriate position, the original architecture of 3-RRR (Figure 4 (a)) are converted. Thus the workspace of the orginal PPM is transformed. The three configurations such as 3-ㅅRP, 3-ㅍR and 3-RPR (Figure 4(a)-(c)) can also establish the hybrid platform to transform the driven layout.

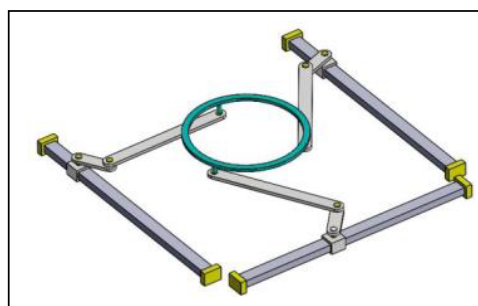

(a)

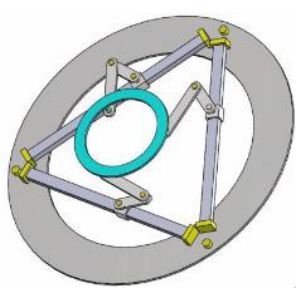

(b)

Figure.5 The parallel hybrid platform: (a) orthogonal layout and (b) triangular layout.

\section{Workspace analysis and simulation}

Workspace is an important characteristic for parallel mechanism. The workspace is defined as the region that consists of a large number of spatial points can be reached by the output point $P$.

Taking the kinematic model of 3-RRR parallel mechanism as representative object, and the motion model is shown in Figure 6. According to Literature [20], if $l_{4}$ is the longest link, $l_{4 \max }>l_{2}>l_{l}$, 
$l_{2}>l_{3}$, and $l_{4 \max }<\sqrt{3} l_{2}+l_{3}$, we derive the conditions of three-crank 3-RRR RPPM, and the following inequality condition should be hold.

$$
2\left(l_{1}+l_{2}\right)-l_{3}<l_{4 \max }<2 l_{2}+l_{3}
$$

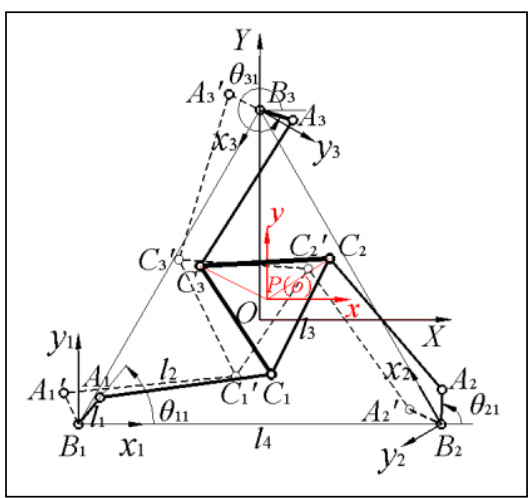

Figure.6 Motion model of a planar 3-DOF parallel mechanism with revolute actuators.

The symmetrical 3-RRR PPM is investigated as in Figure 6, with link lengths $l_{1}=80 \mathrm{~mm}$, $l_{2}=400 \mathrm{~mm}, l_{3}=300 \mathrm{~mm}$, and $l_{4}=890 \mathrm{~mm}$.

According to the kinematics analysis of section 3, we solve the reachable workspace with the help of Matlab software, and the results of the reachable workspace for 3-RRR PPM is shown in Figure 7. The workspace is a symmetrical distribution. The search method is based on the kinematic analysis, which requires much computation for more complex configuration.

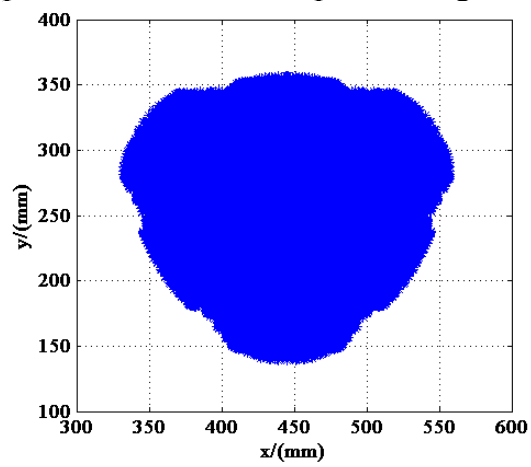

Figure.7 The reachable workspace for 3-RRR PPM with Matlab.

In order to draw the reachable workspace for the RPPM, the superposition method of motion trajectory for each kinematic limb is adopted in this study. The motors of the three kinematic chains $\left(S_{1}, S_{2}\right.$ and $\left.S_{3}\right)$ are actuated separately, and the output trajectories of point $P$ in the moving platform are tracked. There are three steps in the procedure of trajectory simulation.

- The first motor of kinematic chain $S_{1}$ is actuated with appropriate input parameters, but the motor parameters of the other two kinematic chains $S_{2}$ and $S_{3}$ are not actuated and follow the kinematic chain $S_{1}$. Thus the first motion trajectory is drawn.

- Refer to the first step, the other two motion trajectories can be drawn.

- The values of three motion trajectories are got in EXCEL CSV file format. And the three group values are superimposed together in one scatter diagram.

The trajectories of three metamorphic driven layouts for 3-RRR PPM have been simulated using the SolidWorks motion software, as shown in Figure 8. 


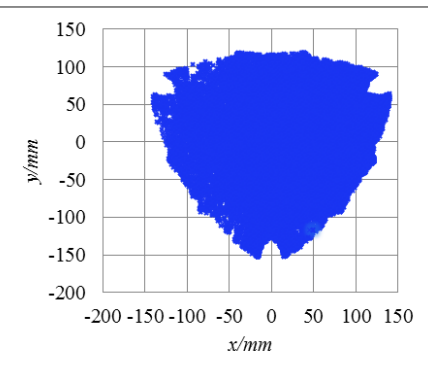

(a)

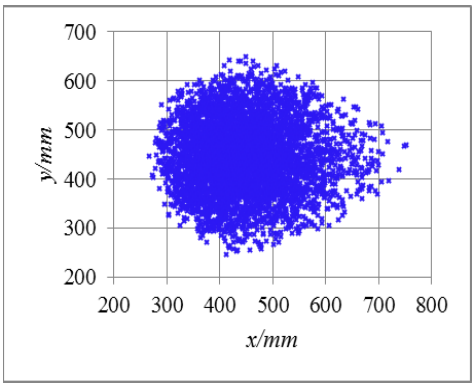

(b)

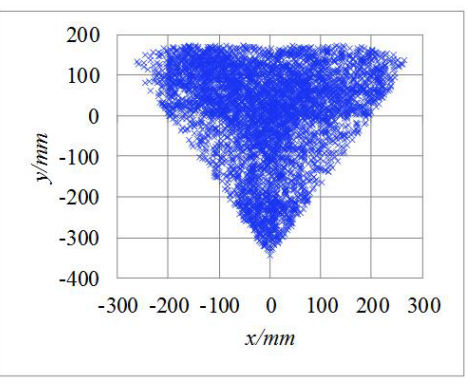

(c)

Figure.8 Reachable workspace of 3-RRR configurations by SolidWorks motion software : (a) original layout, (b) orthogonal layout, and(c) triangular layout.

Compared with Figure 7 and Figure 8(a), the almost identical graphics are obtained with the two methods. As can be seen from Figure 8, the motion trajectories are infinitely close to the boundary of the workspace. From Figure 8(a)-(c), three different workspaces by comparing the boundary coordinates can be found. Otherwise, hybrid platform can expand the workspace of the RPPM.

\section{Summary}

A new full information modeling method has been proposed to the RPPM. By establishing main models of the 3-DOF PPMs, a series of mechanisms configurations have been obtained from the original models. It not only supports the integration parameters modeling between the configuration design and motion analysis but also reconfigurable design of PPMs related to main model.

The superposition method of motion trajectory is adopted in this paper approximate to the real workspace, and avoids complex calculation process. The work has provided a new starting point for further research on reconfigurable mechanisms.

\section{References}

1. K.T. Zhang and J.S. Dai, J Mech Design 137,1 (2015)

2. X. Kong, Mech Mach T 74, 188 (2014)

3. W. Ye, Y. Fang, K. Zhang, S.Guo, Mech Mach T 74,1 (2014)

4. Q. Zeng, K.F. Ehmann, Mech Mach T 71,1(2014)

5. L. Carbonari, M. Callegari, G. Palmieri, M.C. Palpacelli, Mech Mach T 79,173 (2014)

6. D. Zhang, Parallel robotic machine tools(Springer, New York,2010)

7. Z. Bi, L. Wang, Robot CIM 25,591 (2009)

8. Perez R, Molina A, Ramirez-Cadena M, J MANUF SCI 136,213 (2013)

9. W. Yao, J.S. Dai, IND Robot 37,542 (2010)

10. W. Yao, F. Cannella, J.S. Dai, Robot CIM 27,604 (2011)

11. J.S. Dai, Screw Algebra and Li Groups and Lie Algebras( Higher Education Press, Beijing, 2014)

12. W.X. Zhang, X.L. Ding, J.S. Dai, J Mech Eng (in Chinese)49,601(2013)

13. S. Guo, Z.Y. Sun, H.B. Qu, J Mech Eng (in Chinese)51,35(2015)

14. F. Xi, Y. Li, H. Wang,2010 International Conference on Mechatronics and Automation (IEEE, New York,2010)

15. L. Carbonari, M. Callegari, Palmieri G, M.C Palpacelli, Mech Mach T 79,173 (2014)

16. Y.K. Yong, T.F. Lu, Mech Mach T 44,1156 (2009)

17. Z.F. Shao, X.Q. Tang, X. Chen, L. Wang, Chin. J. Mech Eng 22,791 (2009)

18. E. Can, H. Stachel, Mech Mach T 79,29 (2014)

19. B. Bihari, D. Kumar, C. Jha, V.S. Rathorea1, A.K. Dasha, Robotica 34, 738, (2016)

20. R.Q. Li, J.S. Dai, Scie. China Ser. E 52, 3601(2009) 